\title{
Guar gum and hydroxy propyl methylcellulose compressed coated tablets for colonic drug delivery: in vitro and in vivo evaluation in healthy human volunteers
}

\author{
Fahima M. Hashem ${ }^{1}$, Dalia S. Shaker ${ }^{1}$, Mohamed Nasr ${ }^{1, *}$, Ibrahim E. Saad ${ }^{2}$, Reem Ragaey ${ }^{1}$ \\ ${ }^{1}$ Department of Pharmaceutics and Industrial Pharmacy, Faculty of Pharmacy, Helwan University, Cairo, Egypt; \\ ${ }^{2}$ Department of Clinical Oncology and Nuclear Medicine, Faculty of Medicine, Cairo University, Cairo, Egypt.
}

\begin{abstract}
The objectives of the present study are to evaluate guar gum in combination with hydroxy propyl methylcellulose (HPMC) as compression coat for colonic delivery of prednisolone as well as improving the mechanical properties of the compressed coated tablets. The core tablets containing 5 mg prednisolone were compression coated with 125 mg of coating materials consisted of guar gum alone or mixtures of guar gum in combination with different ratios of HPMC. The compressed coated tablets were evaluated for their mechanical properties, in vitro drug release and in vivo performance in human volunteers. The compressed coated tablets with coats containing HPMC exhibited acceptable mechanical properties. In vitro drug release studies in $\mathrm{pH} 7.4$ phosphate-buffered saline medium containing $2 \%$ (w/v) rat caecal content have shown that increase in concentration of HPMC in the prepared coats from $10 \%$ to $20 \%$ resulted in an increase in the release rate. However, further increase in HPMC concentration to constitute $30 \%$ caused a reduction in the release rate. Based on the drug release results, tablets coated with coat consisted of $80 \%$ guar gum and $20 \%$ HPMC were selected for in vivo evaluation. In vivo gamma scintigraphic study on human volunteers using technetium-99m-diethylenetriamine pentaacetic acid as a tracer was performed. The results showed that tablets remained intact in stomach and small intestine, however partial and complete release of the tracer occurred in the colon. In conclusion, guar gum in combination with HPMC would be successfully used as a carrier for drug delivery to the colon.
\end{abstract}

Keywords: Guar gum, hydroxy propyl methylcellulose, compressed coated tablets, colonic drug delivery, prednisolone, gamma scintigraphy

\footnotetext{
* Address correspondence to:

Dr. Mohamed Nasr, Department of Pharmaceutics and Industrial Pharmacy, Faculty of Pharmacy, Helwan University, Cairo, Egypt.

e-mail:m2nasr@yahoo.com
}

\section{Introduction}

Colonic drug delivery has gained increased importance not just for the delivery of drugs for the treatment of local diseases of colon such as irritable bowel syndrome, inflammatory bowel disease (IBD) including Crohn's disease and ulcerative colitis but also for its potential for the delivery of proteins and therapeutic peptides like insulin (1-3).

Colon as a site offers distinct advantages on account of a near neutral $\mathrm{pH}$, a much longer transit time, reduced digestive enzymatic activity, much greater response to absorption enhancers, and the presence of large amounts of enzymes for polysaccharides which were secreted by a large number of colonic bacteria (4). Various systems have been developed for colon-specific drug delivery including covalent linkage of a drug with a carrier, coating with $\mathrm{pH}-$ sensitive polymers, time dependent release systems and enzymatically controlled delivery systems (5). The most convenient approach for site-specific drug delivery to colon is the enzymatically controlled delivery system (6).

Guar gum is a polysaccharide derived from the seeds of Cyamopsis tetragonolobus, of the Leguminosae family. It consists of linear chains of (1-4)- $\beta$-D-mannopyranosyl units with $\alpha$-D-galactopyranosyl units attached by (1-6) linkages. In pharmaceutical formulations, it is used as a binder, disintegrant, suspending agent, thickening agent and stabilizing agent. It was reported previously that guar gum as compression coat is a potential carrier for colonspecific drug delivery (7-10). From the previous studies a coat of considerable thickness of guar gum is usually required to protect the drug loaded in the core tablets, moreover, using guar gum alone in formulation of the compressed coated tablets gave very soft coats (7). In this study, guar gum in combination with hydroxy propyl methylcellulose (HPMC) is used to develop colonic delivery using prednisolone as a model drug. HPMC was used to modify the drug release and improve the mechanical properties of the compressed coated tablets.

The aim of this study is to evaluate a mixture of guar gum and HPMC, in the form of compression coat applied over core tablets for colonic drug delivery. 


\section{Materials and Methods}

\subsection{Materials}

Prednisolone was a gift sample from Al Arabia pharmaceutical Company, Cairo, Egypt. Guar gum was obtained from Sigma-Aldrich, St Louis, MO, USA. HPMC 4000 and Avicel PH101 were obtained from Fluka Biochemika, Buchs, Switzerland. Ethyl alcohol absolute 99\% from the United Company for Chemicals and Medical Preparation, Cairo, Egypt. All other materials used were of pharmacopeial grade.

\subsection{Differential scanning calorimetry (DSC)}

In order to investigate the possible interaction between prednisolone and the polymers used, viz guar gum, HPMC and Avicel PH101, DSC analysis was carried out on pure substances and their physical mixtures in equimolar ratios using the Shimadzu DSC-50 instrument equipped with a computerized data station (Shimadzu, Kyoto, Japan). Samples (4-5 mg) were placed in an aluminum pan and heated at a rate of $10^{\circ} \mathrm{C} / \mathrm{min}$ with indium in the reference pan in an atmosphere of nitrogen to a temperature of $300^{\circ} \mathrm{C}$.

\subsection{Preparation of compression-coated prednisolone tablets}

\subsubsection{Preparation of core tablets}

The core tablets of prednisolone for compression coating were prepared by direct compression technique. Each core tablet $(50 \mathrm{mg}$ ) consisted of $5 \mathrm{mg}$ prednisolone, $44.50 \mathrm{mg}$ Avicel PH101, and $0.5 \mathrm{mg}$ magnesium stearate. The powders were thoroughly mixed and passed through mesh $(149 \mu \mathrm{m})$. The uniformity of mixing was assessed by conducting content uniformity tests on the samples of powder mix. The mixture was compressed into tablets using hydraulic press with an applied force of $3,250 \mathrm{~kg}$ using $4 \mathrm{~mm}$ round concave punches.

\subsubsection{Preparation of compression coated tablets}

The coating materials of $125 \mathrm{mg}$ guar gum alone or mixtures of guar gum in combination with $10 \%, 20 \%$, and $30 \%$ HPMC were used to prepare four coats F1, F2, F3, and F4, respectively. Half the amount of compression coating material was placed in the die cavity followed by carefully centering the core tablet and addition of the remaining coat weight. The coating material was then compressed around the core tablets using hydraulic press at an applied force of 4,000 kg using $8 \mathrm{~mm}$ round concave punches. The prepared tablets were tested for the uniformity of weight, drug content, mechanical properties (hardness and friability) and drug release characteristics.

\subsection{Determination of drug content in tablets}

Ten tablets of each formula were finely powdered; $200 \mathrm{mg}$ of the powder were accurately weighed and transferred to $100 \mathrm{~mL}$ volumetric flasks containing 50 $\mathrm{mL}$ of phosphate buffer $\mathrm{pH}$ 7.4. The flasks were shaken to solubilize the drug. The volume was made up with the buffer to $100 \mathrm{~mL}$, mixed well and allowed to stand for 24 $\mathrm{h}$ to ensure complete solubility of the drug. The solution was centrifuged and $1 \mathrm{~mL}$ of the supernatant liquid was suitably diluted and analyzed for prednisolone content spectrophotometerically at $245 \mathrm{~nm}$.

\subsection{In vitro drug release studies in $0.1 \mathrm{~N} \mathrm{HCl}$ and phosphate buffer, $\mathrm{pH} 7.4$}

The ability of the prepared tablets to retard drug release in the physiological environment of the stomach and small intestine was assessed by conducting drug release studies in simulated stomach and small intestine $\mathrm{pH}$, respectively. Dissolution test was conducted in USP I apparatus at $100 \mathrm{rpm}$ and a temperature of $37^{\circ} \mathrm{C}$. Initial drug release studies were conducted in $700 \mathrm{~mL} 0.1 \mathrm{~N} \mathrm{HCl}$ for $2 \mathrm{~h}$, then $200 \mathrm{~mL}$ of $0.2 \mathrm{M}$ tribasic sodium phosphate was added to the dissolution vessels and $\mathrm{pH}$ was adjusted to $7.4 \mathrm{using}$ $0.1 \mathrm{~N} \mathrm{NaOH}$. Samples were withdrawn at 1, 2, 3, 4, 5, $6,8,10,12,16,20$, and $24 \mathrm{~h}$ time intervals and replaced with an equal volume of fresh media (11). The content of prednisolone in the withdrawn samples was analyzed spectrophotometerically at $245 \mathrm{~nm}$.

\subsection{In vitro drug release studies in presence of rat caecal content}

In order to assess the ability of the prepared tablets to release drug in the physiological environment of the colon, the drug release studies were carried out in $200 \mathrm{~mL}$ of $\mathrm{pH} 7.4$ phosphate-buffered saline (PBS) containing $2 \%(\mathrm{w} / \mathrm{v})$ of rat caecal contents. Approval to carry out the release studies in presence of rat caecal content was obtained from the Animal Ethics Committee of Faculty of Pharmacy, Helwan University. Guidelines of the ethics committee were followed for the studies.

The caecal contents were obtained from Wistar rats weighing 150-200 g, after pre-treatment with oral administration of $2 \mathrm{~mL}$ of $1 \%$ guar gum dispersion in water for 3 days (12). Thirty minutes before starting drug release studies, each rat was killed by spinal traction, after which abdomens were opened, dissected, and immediately transferred to PBS previously bubbled with $\mathrm{CO}_{2}$. The caecal bags were then opened; their contents were individually weighed, homogenized, and then suspended in PBS to give the desired concentration of $2 \%(\mathrm{w} / \mathrm{v})$ of caecal content. As the caecum is naturally anaerobic, all these operations were carried out under $\mathrm{CO}_{2}$.

Drug release studies in the caecal content were carried out on tablets previously subjected to $5 \mathrm{~h}$-exposure to 
conditions mimic the stomach and the small intestine. The obtained tablets were then placed in $200 \mathrm{~mL}$ of the dissolution medium (PBS, $\mathrm{pH}$ 7.4) containing $2 \%(\mathrm{w} / \mathrm{v})$ rat caecal content. The release studies were performed using USP I apparatus at $100 \mathrm{rpm}$ and a temperature of $37^{\circ} \mathrm{C}$ with continuous $\mathrm{CO}_{2}$ supply into the dissolution media. At specific time intervals, samples were withdrawn and replaced with fresh medium. The experiment was continued up to $24 \mathrm{~h}$. The withdrawn samples were filtered through $0.45 \mu \mathrm{m}$ membrane filter and analyzed for drug content at 245 nm spectrophotometerically.

\subsection{In vitro release kinetics mechanisms}

In order to determine drug release mechanism from the prepared tablets, the release kinetic data were analyzed according to Korsmeyer-Peppas release model (13) given by the following equation:

$$
\mathrm{M}_{\mathrm{t}} / \mathrm{M}_{\infty}=\mathrm{Kt}^{\mathrm{n}}
$$

where $M_{t}$ is the amount of drug released at time $t ; M_{\infty}$ is the amount of drug released at infinite time; $\mathrm{K}$ is the kinetic constant related to the structural and geometric characteristics of the drug delivery system (tablet); and $\mathrm{n}$ is the release exponent indicative of the release mechanism. The $n$ values used for elucidation of drug release mechanism from the tablets were determined from log cumulative percentage of drug release versus log time plots. Values of $\mathrm{n}$ near 0.5 indicate predominantly diffusion control and of 1.0 correspond to zero-order release. Another analysis mechanism was used considering that drug release in swellable matrices depends on two processes, drug diffusion into the swollen polymer and matrix swelling due to approximate contribution of the diffusion and relaxation mechanisms. This was carried out by fitting the data to the model proposed by Peppas and Sahlin (14) given by the following equation:

$$
\mathrm{M}_{\mathrm{t}} / \mathrm{M}_{\infty}=\mathrm{K}_{1} \mathrm{t}^{\mathrm{m}}+\mathrm{K}_{2} \mathrm{t}^{2 \mathrm{~m}}
$$

where $\mathrm{K}_{1}$ and $\mathrm{K}_{2}$ are obtained from non linear regression curve fitting of the release data using GraphPad prism 4 (GraphPad Software, San Diego, CA, USA). When $\mathrm{K}_{1}>\mathrm{K}_{2}$, the release is mainly controlled by diffusion, and when $\mathrm{K}_{2}>\mathrm{K}_{1}$, the release is mostly due to matrix swelling. When $\mathrm{K}_{1}$ is nearly equal to $\mathrm{K}_{2}$, the release is a combination of diffusion and polymer relaxation (15).

\subsection{Preparation of labeled tablets for in vivo scintigraphic studies}

The core tablet (average weight $50 \mathrm{mg}$ ) for in vivo scintigraphic study consists of sodium chloride (20 $\mathrm{mg}$ ), Avicel (29.50 mg) and magnesium stearate (0.5 $\mathrm{mg}$ ). Sodium chloride was used as filler and 5 millicuri of ${ }^{99 \mathrm{~m}} \mathrm{Tc}$-diethylenetriamine pentaacetic acid (DTPA), a radiolabelled material, was adsorbed on it. ${ }^{99 \mathrm{~m}} \mathrm{Tc}$ DTPA was prepared by radiolabelling DTPA with sodium pertechnetate solution. Sodium chloride was dissolved in this solution, evaporated to dryness. The resultant powder was then mixed with the remaining excipients and compressed into tablets using $4 \mathrm{~mm}$ round concave punches. The core tablets were then compress-coated with $125 \mathrm{mg}$ of the coating material F3 (80\% guar gum and 20\% HPMC).

\subsection{In vivo scintigraphic studies}

The study was approved by the University Protection of Human Subjects Committee, and the protocol complies with the declarations of Helsinki and Tokyo for humans. Six healthy male volunteers participated in this study. After overnight fasting, each volunteer orally swallowed the prepared radiolabelled tablets. The tablets were scanned using a PHILIPS AXIS dual head gamma camera (Phillips Medical System, Cleveland, OH, USA). Anterior and posterior images were taken immediately after tablet administration and after $0.5,1,1.5,2,4,8$, 12 , and $24 \mathrm{~h}$.

\subsection{Statistical analysis}

Student $t$-test was used to test the differences between the calculated parameters using SPSS Statistical Package, Version 10 (IBM SPSS, Chicago, IL, USA). Statistical differences yielding $p<0.05$ were considered to be significant.

\section{Results and Discussion}

\subsection{DSC study}

DSC thermograms revealed that prednisolone has a single sharp characteristic, endothermic melting peak at $240.94^{\circ} \mathrm{C}$ (Figure 1, trace A). The sharp endothermic peak reflects the pure crystalline state of the drug. The sharp endothermic peak of the drug was observed at the same melting temperature in case of its physical mixtures with Avicel, guar gum and HPMC but shortened due to the dilution factor (Figure 1). These results demonstrated that prednisolone did not interact with the chosen additives.

\subsection{Physical properties of tablets}

All the prepared tablets met the USP requirements for weight variation, hardness, and drug content (Table 1). The friability of the prepared tablets was within the compendial limits except F1 coated with $100 \%$ guar gum which showed high friability percentage $(2.20 \%)$ that exceeded the pharmacopeial limitation. Therefore, F1 was excluded from any further evaluations. 


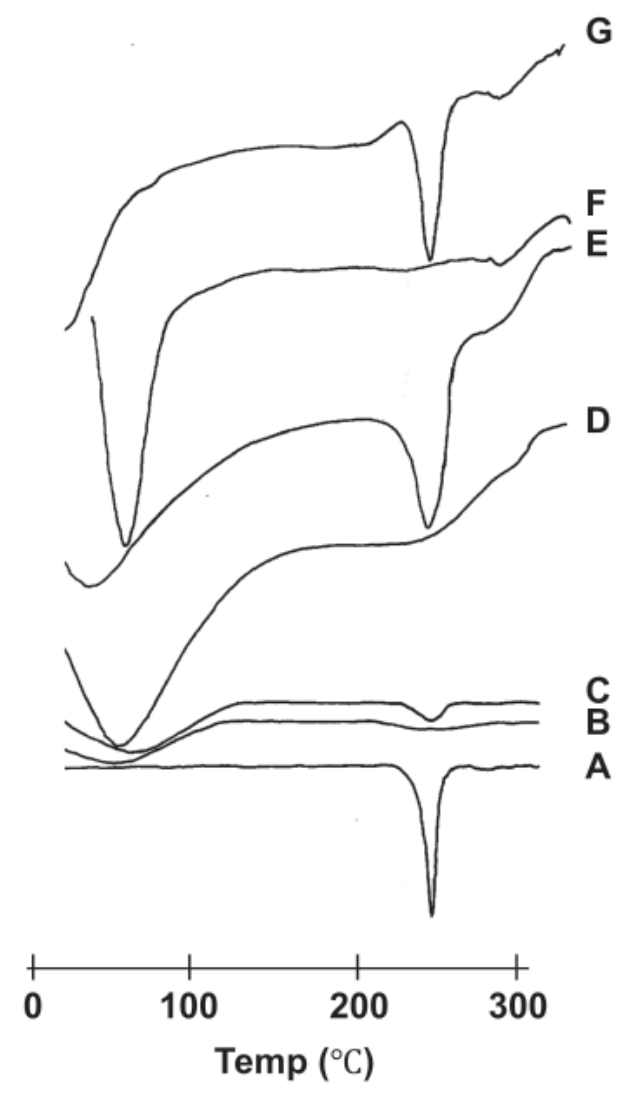

Figure 1. DSC thermograms. A, prednisolone; B, Avicel; C, prednisolone-Avicel physical mixture; D, guar gum; E, prednisolone-guar gum physical mixture; F, HPMC; and G, prednisolone-HPMC physical mixture.

Table 1. Physical properties of the compressed coated tablets

\begin{tabular}{lcccc}
\hline Formulations & $\begin{array}{c}\text { Weight } \\
(\mathrm{mg} \pm \text { S.D. })\end{array}$ & $\begin{array}{c}\text { Hardness } \\
\left(\mathrm{kg} / \mathrm{cm}^{2} \pm \text { S.D. }\right)\end{array}$ & $\begin{array}{c}\text { Friability } \\
(\%)\end{array}$ & $\begin{array}{c}\text { Drug content } \\
(\% \pm \text { S.D. })\end{array}$ \\
\hline F1 & $177.00 \pm 1.50$ & $4.50 \pm 0.44$ & 2.20 & $4.90 \pm 0.15$ \\
F2 & $176.00 \pm 1.13$ & $5.30 \pm 0.48$ & 0.42 & $4.94 \pm 0.10$ \\
F3 & $175.40 \pm 1.76$ & $5.60 \pm 0.51$ & 0.32 & $5.00 \pm 0.05$ \\
F4 & $176.00 \pm 1.51$ & $6.10 \pm 0.62$ & 0.25 & $4.98 \pm 0.11$ \\
\hline
\end{tabular}

\subsection{In vitro release studies}

The mean drug release from the tablets F2, F3, and F4 after the first $5 \mathrm{~h}$ was $2.67 \pm 0.15 \%, 6.43 \pm 0.54 \%$, and $8.27 \pm 0.25 \%$, respectively. At the end of $24 \mathrm{~h}$, the mean $\%$ drug release was $37.7 \pm 2.7 \%, 47.2 \pm 2.2 \%$, and 31.2 $\pm 2.0 \%$, respectively, in PBS medium (Figure $2 \mathrm{~A}$ ). While in rat caecal medium, after $24 \mathrm{~h}$, the mean $\%$ drug release was increased to $91.0 \pm 4.1 \%, 97.2 \pm 2.2 \%$ and 82.5 $\pm 4.1 \%$, respectively (Figure $2 \mathrm{~B}$ ). The maximum drug release after $24 \mathrm{~h}$ in rat caecal medium was significantly higher $(p<0.05)$ in comparison with the drug release in control medium. This can be explained as the release of prednisolone in the physiological environment of colon is due to microbial degradation of guar gum (4).

The addition of HPMC to constitute $10 \%$ to $20 \%$ of the coat (F2 and F3, respectively) caused a significant increase $(p<0.05)$ in the mean $\%$ drug release from 37.7
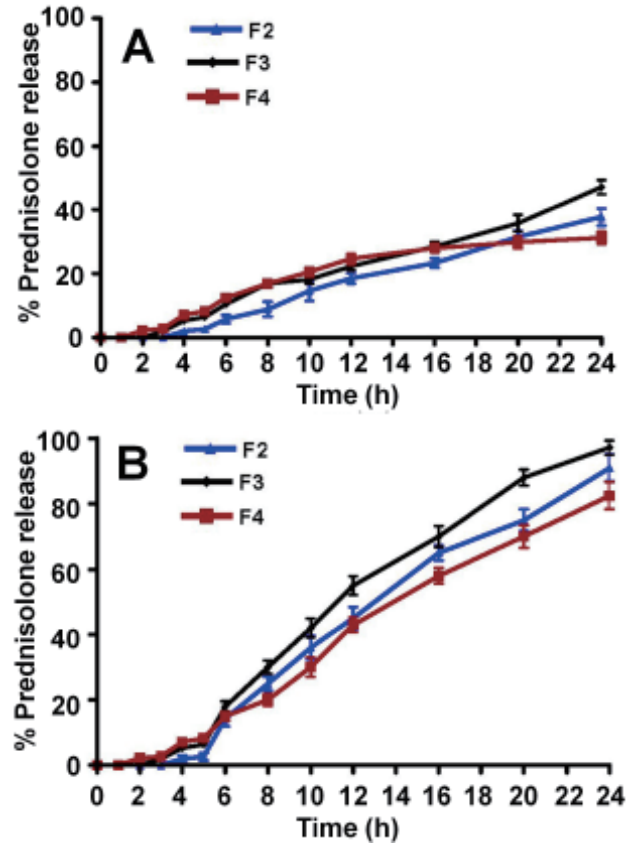

Figure 2. Release profiles of prednisolone from compressioncoated tablets F2, F3, and F4. (A) Release profiles in $0.1 \mathrm{~N}$ $\mathrm{HCl}$ for $2 \mathrm{~h}$ and phosphate buffer (pH 7.4) till the end of $24 \mathrm{~h}$. (B) Release profiles in $0.1 \mathrm{~N} \mathrm{HCl}$ for $2 \mathrm{~h}$, phosphate buffer $(\mathrm{pH} 7.4)$ for another $3 \mathrm{~h}$, and PBS containing $2 \%(\mathrm{w} / \mathrm{v})$ rat caecal content till the end of $24 \mathrm{~h}$.

$\pm 2.7 \%$ to $47.2 \pm 2.2 \%$ after $24 \mathrm{~h}$ in the physiological environment simulating the stomach and small intestine and from $91.0 \pm 4.1 \%$ to $97.7 \pm 2.2 \%$ and in rat caecal medium. The increase in drug release could be explained due to HPMC creates a porous structure of the coat and consequently increases guar gum leaching and drug release. However, further increase in HPMC percent to constitute $30 \%$ of the compression coat (F4) caused a reduction in gum leaching, with a consequent decrease in drug release as shown in Figure 2A. These results are well correlated with previous reports $(16,17)$ which suggested that higher concentrations of HPMC would reduce the free water volume and increase the viscosity of the coat causing a reduction in the polymer leaching and subsequent reduction in drug release. Based on the previous results, F3 was selected for further in vivo evaluation since the cumulative percentage of drug released at the end of $5 \mathrm{~h}$, which is the expected time for the arrival of the dosage form in the colon, was found to be $6.43 \pm 0.54 \%$ and almost complete drug release was achieved after $24 \mathrm{~h}$.

The kinetics of prednisolone release from the prepared tablets was studied by applying the Korsmeyer model to the release data up to $60 \%$ of prednisolone. The release kinetic parameters are listed in Table 2. Increase of the HPMC content in the coat of F2, F3, and $\mathrm{F} 4$ results in exponents $\mathrm{n}$ values of $1.53,1.40$, and 1.45 , respectively, which markedly exceed the value of 0.5 corresponding to diffusion controlled release and furthermore together with the good fitting of the zeroorder model indicate significant contribution of erosion. 
Table 2. Fitting of release kinetic models to prednisolone release data

\begin{tabular}{|c|c|c|c|c|c|c|c|}
\hline \multirow{2}{*}{ Formulations } & \multicolumn{2}{|c|}{ Zero-order } & \multicolumn{2}{|c|}{ Korsmey ermodel* } & \multicolumn{3}{|c|}{ Peppas-Sahlin model } \\
\hline & $\mathrm{R}^{2}$ & $\mathrm{~K}_{\mathrm{o}}\left(\% \mathrm{~h}^{-1}\right)$ & $\mathrm{R}^{2}$ & $\mathrm{n}$ & $\mathrm{R}^{2}$ & $\mathrm{~K}_{1}\left(\% \mathrm{~h}^{-0.45}\right)$ & $\mathrm{K}_{2}\left(\% \mathrm{~h}^{-0.9}\right)$ \\
\hline $\mathrm{F} 2$ & 0.985 & 4.475 & 0.987 & 1.53 & 0.985 & -12.23 & 8.37 \\
\hline F3 & 0.982 & 4.832 & 0.979 & 1.40 & 0.983 & -11.11 & 8.67 \\
\hline F4 & 0.992 & 3.902 & 0.985 & 1.45 & 0.993 & -9.05 & 7.06 \\
\hline
\end{tabular}

* Release exponent evaluated for $<60 \%$ released drug.
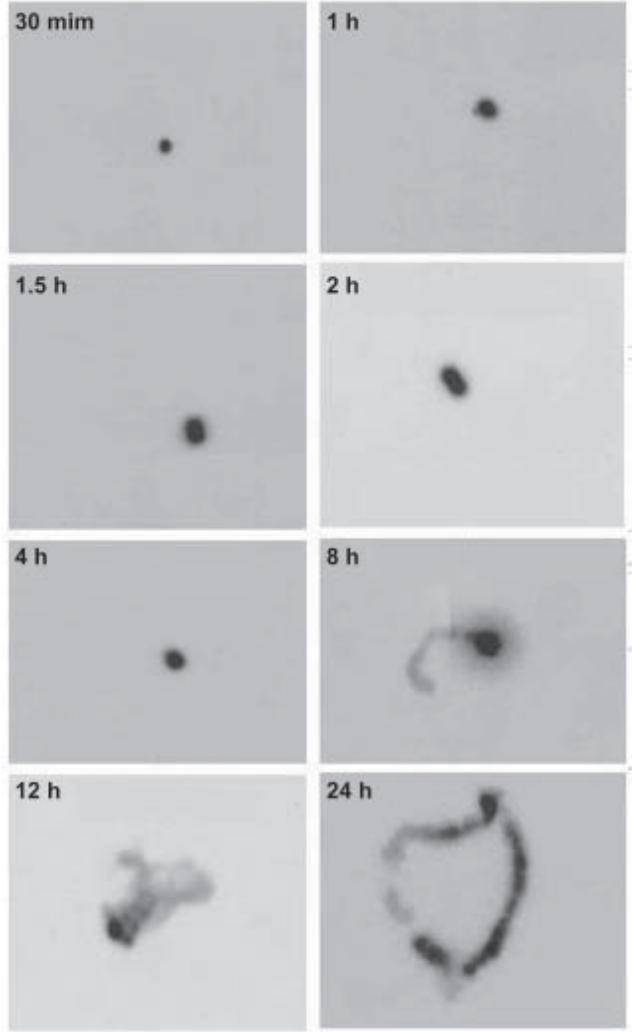

Figure 3. A representative gamma scintigraphs in one volunteer. Tablets remained intact in stomach after $0.5,1$, and $1.5 \mathrm{~h}$; in small intestine after 2 and $4 \mathrm{~h}$. Tablets partially disintegrated in colon after 8 and $12 \mathrm{~h}$, and completely disintegrated and distributed as the tracer throughout the colon after $24 \mathrm{~h}$.

The higher values of $\mathrm{n}$ would be a consequence of a plasticization process in the gel layer arising from a reduction of the attractive forces among polymeric chains that increases the mobility of macromolecules (18). Further analysis by Peppas and Sahlin model showed higher values of the relaxation constant $\mathrm{K}_{2}$, compared with the diffusion constant $\mathrm{K}_{1}$, combined with the low solubility of prednisolone, reflect the prevalence of the erosion as a mechanism for drug release versus swelling mechanism.

\subsection{In vivo $\gamma$-scintigraphic studies}

From the images taken at regular time intervals in all volunteers, the observed time for initiation of tablet disintegration and distribution of the tracer through gastrointestinal tract were closely similar. Figure 3 shows a group of representative images for initiation of disintegration of the tablets and distribution of the traces in gastrointestinal tract of one volunteer. From the images, it was found that the tablets remained intact in stomach (30 and $60 \mathrm{~min}$ ) and small intestine ( 2 and $4 \mathrm{~h}$ ). On entering the colon, the tablets began to release the tracer due to partial degradation of the coat ( 8 to $12 \mathrm{~h}$ ) and finally the uniform distribution of the tracer along the entire colon after $24 \mathrm{~h}$. These results showed that a coat consists of a mixture of $80 \%$ guar gum and 20\% HPMC would successfully prevent the release of prednisolone in the stomach and small intestine. However, on arrival to the colon, the tablets started their degradation after $8 \mathrm{~h}$ and were completely disintegrated after $24 \mathrm{~h}$ as evident from the distribution of the tracer in the different segments of the colon as shown in Figure 3.

\section{Conclusion}

Based on drug release in the colon as well as in vivo gamma scintigraphic study, compressed coated tablets with mixture of $80 \%$ guar gum and 20\% HPMC could produce a successful drug targeting to the colon with minimal amount released in the gastrointestinal tract.

\section{References}

1. Chourasia MK, Jain SK. Pharmaceutical approaches to colon targeted drug delivery systems. J Pharm Pharm Sci. 2003; 6:33-66.

2. Yang L, Chu JS, Fix JA. Colon-specific drug delivery: New approaches and in vitro/in vivo evaluation. Int $\mathrm{J}$ Pharm. 2002; 235:1-15.

3. Ugurlu T, Turkoglu M, Gurer US, Akarsu BG. Colonic delivery of compression coated nisin tablets using pectin/ HPMC polymer mixture. Eur J Pharm Biopahrm. 2007; 67:202-210.

4. Sinha VR, Kumria R. Polysaccharides in colon-specific drug delivery. Int J Pharm. 2001; 224:19-38.

5. Leopold CS. Coated dosage forms for colon-specific drug delivery. Pharm Sci Technolo Today. 1999; 2:197-204.

6. Liu L, Fishman ML, Kost J, Hicks KB. Pectin-based systems for colon-specific drug delivery via oral route. Biomaterials. 2003; 24:3333-3343.

7. Krishnaiah YS, Satyanarayana S, Rama Prasad YV, Narasimha Rao S. Evaluation of guar gum as a compression coat for drug targeting to colon. Int J Pharm. 1998; 171:137-146.

8. Krishnaiah YS, Satyanarayana S, Prasad YV. Studies 
of guar gum compression-coated 5-aminosalicylic acid tablets for colon-specific drug delivery. Drug Dev Ind Pharm. 1999; 25:651-657.

9. Krishnaiah YS, Bhaskar Reddy PR, Satyanarayana V, Karthikeyan RS. Studies on the development of oral colon targeted drug delivery systems for metronidazole in the treatment of amoebiasis. Int J Pharm. 2002; 236:43-55.

10. Yehia SA, Elshafeey AH, Sayed I, Shehata AH. Optimization of budesonide compression-coated tablets for colonic delivery. AAPS PharmSciTech. 2009; 10:147-157.

11. He W, Du Q, Cao DY, Xiang B, Fan LF. Study on colonspecific pectin/ethyl cellulose film-coated 5-flurouracil pellets in rats. Int J Pharm. 2008; 348:35-45.

12. Rubinstein A, Radai R, Ezra M, Pathak S, Rokem JS. In vitro evaluation of calcium pectinate: A potential colon-specific drug delivery carrier. Pharm Res. 1993; 10:258-263.

13. Korsmeyer RW, Gurney R, Doelker E, Buri P, Peppas NA. Mechanism of solute release from porous hydrophilic polymers. Int J Pharm. 1983; 15:25-35.
14. Peppas NA, Sahlin JJ. A simple equation for description of solute release. III. Coupling of diffusion and relaxation. Int J Pharm. 1989; 57:169-172.

15. Kim H, Fassihi R. Application of a binary polymer system in drug release rate modulation. 1. Characterization of release mechanism. J Pharm Sci. 1997; 86:316-322.

16. Frohooff Hulsmann MA, Lippold BC, McGinity JW. Aqueous ethyl cellulose dispersion containing plasticizers at different water solubility and HPMC as coating material for diffusion pellets II: Properties of sprayed films. Eur J Pharm Biopahrm. 1999; 48:67-75.

17. Lindstedt B, Sjöberg M, Hjärtstam J. Osmotic pumping release from KCL tablets coated with porous and nonporous ethyl cellulose. Int J Pharm. 1991; 67:21-27.

18. Llabolt JM, Manzo RH, Allemandi DA. Drug release from carbomer:carbomer sodium salt matrices with potential next term use as previous term mucoadhesive drug delivery system. Int J Pharm. 2004; 276:59-66.

(Received November 02, 2010; Revised February 10, 2011; Re-revised April 12, 2011; Accepted April 14, 2011) 